\title{
interview
}

\section{Ultrafast nanoprobing}

\author{
Imaging the transient carrier dynamics in semiconductors at both high temporal and spatial resolution \\ has long been a goal for solid-state scientists. Hidemi Shigekawa from the University of Tsukuba in Japan \\ told Nature Photonics how his team accomplished this feat.
}

Tell us about the motivation behind your research.

The advancement of ultrashort-pulse laser technology has opened the door to the world of ultrafast phenomena. Optical pump-probe measurements, for example, have made it possible to observe ultrafast dynamics in the femtosecond range, but with spatial resolutions limited by the wavelength of light used. In contrast, scanning tunnelling microscopy (STM) can directly image atomic-scale structures, but with a low temporal resolution of less than $100 \mathrm{kHz}(10 \mu \mathrm{s})$. Thus, since the invention of STM in 1981, the idea of combining time-resolved tunnelling current measurement with ultrashort-pulse laser technology to simultaneously obtain ultimate spatial and temporal resolution has been a very attractive prospect.

What were the challenges in combining STM and photo-excitation? Lock-in detection - a technique that improves the signal-to-noise ratio by measuring the tunnelling current only at a particular frequency - is generally employed while the laser used for the photo-excitation is modulated. The problem is that when the laser intensity is modulated, the displacement current induced by the thermal expansion and shrinking of the STM tip and sample significantly increases the background noise. A distance variation of just $0.1 \mathrm{~nm}$ changes the tunnelling current by one order of magnitude. Furthermore, the large background fluctuates with the unavoidable small fluctuations in laser intensity that occur at low frequencies.

How did you get around the problem? We adopted delay-time modulation as the reference signal for lock-in detection, instead of laser-intensity modulation, to solve the noise problem. The delay-time modulation is controlled by a pulse-picker consisting of an ultrafast Pockels cell that selectively transmits pulses from a sequence of laser pulses. In essence, $800 \mathrm{~nm}$ ultrashort laser pulses with pulse widths of $140 \mathrm{fs}$ are divided into two with a certain time delay to form a train of pulse pairs.
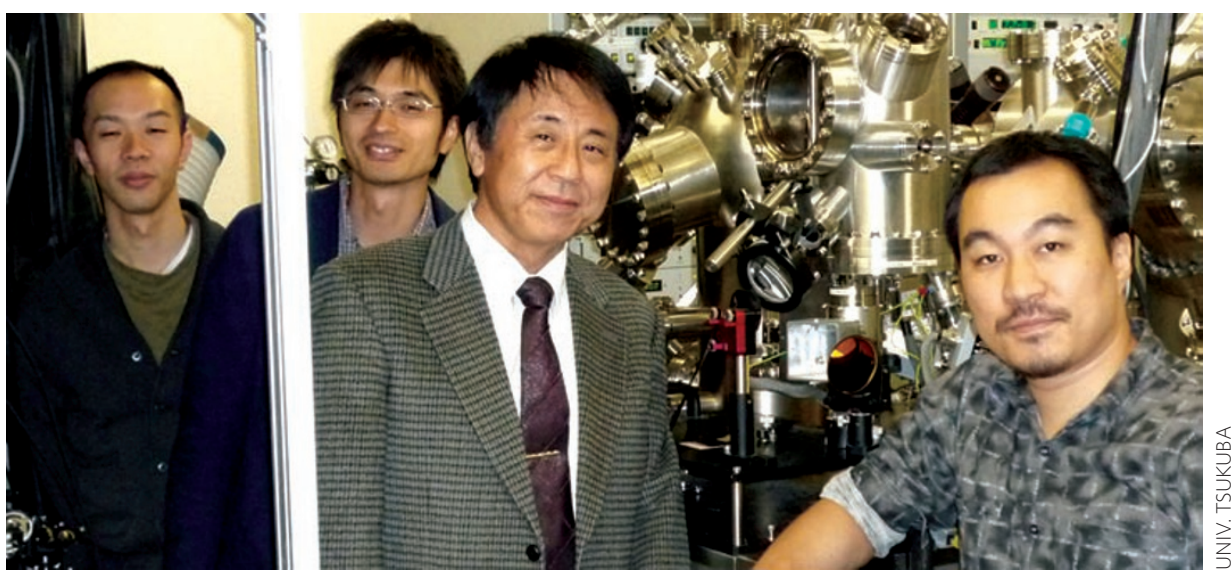

From left to right: Shoji Yoshida, Yasuhiko Terada, Hidemi Shigekawa and Osamu Takeuchi, with their experimental set-up.

This paired-pulse train is then used to excite the sample below the STM tip. The optical pulses create an excited state in the sample and result in a rapid change in a physical quantity such as the photocarrier density, which often gives rise to changes in the raw tunnelling current. Depending on the time delay, the second excitation in the physical quantity will have a different intensity, and hence a different tunnelling current. Thus, by measuring the tunnelling current as a function of the delay time, we demonstrated that the relaxation dynamics of the excited state in the target material can be probed by STM at the resolution of the pulse width.

\section{What have you achieved?}

The control of delay-time modulation using a pulse-picking technique has enabled the visualization of transient carrier dynamics in nanometre-scale structures with a wide range of lifetimes - a technique that has long been desired. We have observed the carrier dynamics of various semiconductor samples such as GaNAs, $\mathrm{GaAs}$ and low-temperature-grown GaAs. The obtained results are consistent with the recombination lifetimes determined from conventional optical pump-probe measurements. We have also directly analysed the recombination of excited carriers via the gap states associated with a cobalt nanoparticle/GaAs structure in real space. Through the site dependence of the decay time on the tunnelling current injected from the STM tip, we were able to image the hole capture rate at the gap states on the nanoscale for the first time. Electrically controlling the delay time allows a large and rapid lock-in modulation to be achieved, providing a signal-tonoise ratio that is 100 times better than if mechanical mirrors were used.

\section{What are the implications of} your findings?

As structures shrink in size, small differences in electronic properties have an increasingly important effect on their macroscopic behaviour. For instance, fluctuations in the distribution of a few dopant materials can govern the properties of macroscopic functions. The ability to directly observe and control ultrafast dynamics on the nanoscale is of great importance for the continued advancement of this field. For further advances, a method of exploring the transient dynamics of the local quantum functions in organized small structures is eagerly desired. Our microscopy method is expected to be useful in this respect.

\section{INTERVIEW BY RACHEL WON}

Hidemi Shigekawa and co-workers have an Article on nanoscale pump-probe microscopy on page 869 of this issue. 